\title{
Chronic Cerebrospinal Venous Insufficiency in Multiple Sclerosis: The Hydrostatic-Immune Paradigm and the Flow Cytometry as a Diagnostic Tool
}

\author{
Tsamopoulos $\mathrm{NG}^{1^{*}}$, Kalodimou VE ${ }^{2}$, and Vlachos $\mathbf{S}^{3}$ \\ ${ }^{1}$ Mediterraneo Hospital, Department of Interventional Neuroradiology, Athens, Greece
}

${ }^{2}$ IASO Maternity \& Research Hospital, Department of Flow Cytometry-Research \& Regenerative Medicine, Athens, Greece

${ }^{3}$ Department of Anesthesiology, Naval and Veterans' Hospital of Athens, Greece

"Corresponding author: N.G. Tsamopoulos, MD, PhD Mediterraneo Hospital - Department of Interventional Neuroradiology, 8-12 llias str. Glyfada, 166 75 Greece, Tel: +302109117010, E-mail: tsamopoulos@gmail.com

Received date: Mar 21, 2014, Accepted date: Apr 22, 2014, Published date: Apr 26, 2014

Copyright: (c) 2014 Tsamopoulos NG, et al. This is an open-access article distributed under the terms of the Creative Commons Attribution License, which permits unrestricted use, distribution, and reproduction in any medium, provided the original author and source are credited.

\begin{abstract}
In recent years, chronic cerebro-spinal venous insufficiency (CCSVI) has been associated with multiple sclerosis (MS). Balloon angioplasty of the affected veins (internal jugulars, azygos) has been proposed as a treatment method, with controversial results. The conflict is based on how a primarily immune disease can be affected by a primarily hydrostatic condition and its reversal. In our paper we briefly review novel paradigms in multiple sclerosis pathogenesis and propose a mechanism by which CCSVI could theoretically lead to blood brain barrier disruption, altered neuronal microenvironment, astrocyte and oligodendrocyte loss and demyelination. Altered antigen transfer to regional lymph nodes, affecting antigen presentation and processing could also contribute, affecting the sensitive balance between tolerance and immunity. Thus, a combined hydrostatic-immune paradigm of MS emerges, which may explain the potential role of CCSVI in MS pathogenesis and provide a theoretical framework for future research.
\end{abstract}

Keywords: Cervical lymph nodes; Chronic cerebrospinal venous insufficiency (CCSVI); Immunity; Multiple sclerosis; Venous insufficiency; Flow cytometry

\section{Introduction}

Multiple sclerosis (MS) is an inflammatory demyelinating disease of the central nervous system (CNS). Subjects 20-40 years old are affected in a relapsing-remitting, secondary progressive or primary progressive pattern. Although the clinical course of the disease is variable, it usually leads to progressive deterioration and severe disability [1]. Both genetic and environmental factors have been implicated in the pathogenesis [2]. The precise cause remains largely unknown, but there is evidence of a myelin-antigen-specific, Th1-mediated immune process resulting in macrophage-mediated demyelination, axonal loss and gliosis [3].

\section{The vascular controversy}

In the last years, a "vascular hypothesis" initially proposed by Charcot [4] has reemerged, causing heated debates among physicians and huge expectations among patients.

Zamboni et al. [5] combined transcranial and extracranial color Doppler ultrasonography to examine internal jugular, vertebral, deep cerebral, and azygous veins and evaluate 5 parameters that assess venous blood flow and vessel anatomy. A significant correlation between MS and the presence of 2 or more criteria of venous outflow abnormalities was reported. This led the authors to a highly criticized hypothesis that chronically impaired venous drainage of the CNS (termed "chronic cerebrospinal venous insufficiency", CCSVI) may trigger the initiation of an inflammatory process which eventually leads to MS $[6,7]$.
Attempts to treat CCSVI by venous angioplasty and/or stenting, a technique termed "liberation procedure", have generated conflicting results [8], adding to the general confusion. Published studies so far display remarkable heterogeneity regarding study protocol and patient recruitment, making comparisons and evidence-based recommendations impossible. This is probably due to poor consensus on terminology and sonographic criteria as well as lack of a standardized methodology regarding patient selection and procedural technique [9].

\section{Newer insights into MS pathogenesis}

In a study of autopsy-derived MS tissue, Barnett et al. [10] reported that, within newly forming lesions, oligodendrocyte apoptosis and intramyelinic edema was accompanied by active phagocytic demyelination. The latter occurred in the absence of contactdependent, cell-mediated immunity, suggesting that macrophage activity may be of a scavenger-like nature, in response to phagocytic ligands on apoptotic oligodendrocytes [11]. Whether oligodendrocyte death and demyelination unmask an autoantigen which causes autoimmunity, or another factor induces both apoptosis and a systemic inflammatory response, is not known [11]. Either way, according to the paradigm proposed by Barnett et al, a population of microglia differentiates into myeloid-like dendritic cells. These migrate to the cervical lymph nodes (CLNs) and prime the differentiation of naïve $\mathrm{T}$ cells towards a central memory and effector phenotype. Such CCR7+CD68+MHCII+ dendritic cells have been observed in CLN tissue [12,13], although their precise ancestry is controversial [14]. Th1 lymphocytes then enter the CNS and are reactivated by antigen-presenting cells (APCs), amplifying the inflammatory response and creating a vicious, ultimately selfsustainable cycle. This temporal diversification of the immune process would explain the heterogeneity of MS lesions observed by Lucchinetti 
Citation: Tsamopoulos NG, Kalodimou VE, and Vlachos S (2014) Chronic Cerebrospinal Venous Insufficiency in Multiple Sclerosis: The Hydrostatic-Immune Paradigm and the Flow Cytometry as a Diagnostic Tool. J Mult Scler 1: 103. doi:10.4172/jmso.1000103

Page 2 of 7

et al. [15] and reconcile observed histopathologic differences between active and chronic lesions.

\section{The hydrostatic approach}

On a more macroscopic scale, MS has been associated with diffuse brain hypoperfusion [16] and decreased cerebral metabolism [17] even in the initial stages of the disease [18]. Using dynamic susceptibility contrast-enhanced magnetic resonance imaging, Law et al. [19] found significantly decreased cerebral blood flow $(\mathrm{CBF})$ and significantly prolonged mean transit time throughout the normal appearing white matter (NAWM) in patients with relapsing- remitting MS. Adhya et al. [20] also found significantly decreased CBF and cerebral blood volume (CBV) in NAWM regions of patients with either relapsingremitting or primary progressive MS compared with healthy subjects.

These findings indicate that globally decreased white matter perfusion may be common in all subtypes of the disease [16]. Blood supply to the grey matter appears to be also compromised [21] either by the same mechanism affecting white matter or as a result of a dissociation between the cerebral cortex and subcortical structures due to the white matter damage. It is not clear whether hypoperfusion in MS is of a primary, vascular aetiology or secondary to reduced metabolic demand from axonal degeneration. Diffusion tensor imaging studies by Saindane et al. [22] point against a secondary insult, while, on a microscopic level, the absence of structural perivascular abnormalities indicates that hypoperfusion of the white matter in MS has a functional vascular origin [23].

Astrocytes occupy a significant proportion of the surface of intracerebral blood vessels and are involved in regulation of vessel diameter and CBF $[24,25]$. In MS white matter lesions and NAWM, astrocytes were found to be deficient in $\beta 2$-adrenergic receptors [26]. Decreased cAMP formation due to impaired $\beta 2$ signaling may lead to vasoconstriction trough several mechanisms [16], implying a key role for astrocyte dysfunction in MS hypoperfusion. The cause of astrocyte dysfunction has not been elucidated yet, but spatial correlation of lesions and blood vessels may provide a clue.

Lightman et al., studying the frequency of retinal vascular abnormalities in patients presenting with acute optic neuritis who went on to develop MS later, noted that “... the presence of perivenular abnormalities in a region free of myelin and oligodendrocytes provides evidence that the vascular changes in MS can occur independently of contiguous demyelination, and may be the primary event in the formation of new lesions" [27].

In initial stages of MS, plaques appear as thin, linear periventricular white matter lesions (Dawson's fingers), oriented around the long axis of central veins [28]. Ultra-high-field 7T MRI studies showed that the majority of these lesions are associated with centrally coursing veins $[29,30]$. The close proximity of evolving plaques to venules has been demonstrated histologically in human brain tissue [31,32] showing perivascular cuffs, intramural fibrinoid accumulation, haemosiderin deposition and collagenised thickened vessel walls that were not seen in control subjects [33]. These histological findings are similar to those described in the case of chronic periphlebitis retinae, suggesting a common pathogenetic mechanism and implying that periphlebitis may be an initial event in plaque formation [34].

Another important finding in MS patients early in the course of the disease is evidence of diminished venous vasculature [35]. According to Zivadinov et al. [36], decreased venous vasculature in brain parenchyma of MS patients is strongly related to concomitant presence and severity of extracranial venous stenoses.

So, altered cerebral vasculature and the inflammatory process underlying MS may share common pathophysiological mechanisms [37]. This relationship is still poorly understood but may involve production and release of soluble substances with vasoactive properties (NO, substance P, Interleukin-1) [38,39] and cytokines (TNF-a) [40]. Changes in neuronal homeostasis due to blood brain barrier (BBB) disruption have also been implicated [41]. In fact, defective endothelial tight junctions (TJ) have been found to coincide with microglial activation in active lesions and NAWM [42]. According to the authors: "The restriction of "putatively open" junctions to venules in active lesions and microscopically abnormal NAWM and the absence of similar alterations in chronic plaques may point to a specific mechanism of reversible junctional disruption restricted to such vessels, and related to the latter's involvement in the diapedesis of inflammatory cells". In other words, “... altered endothelial cell function and might be the common final pathway leading to changes in perfusion and BBB leakage" [37]. The cause of endothelial malfunction is not clear but one theory suggests that venous stenoses may contribute [43].

Abnormal venous outflow, as described in patients with CCSVI, is not only due to downstream anatomical restrictions in blood flow (abnormal jugular valve, stenosis etc). The effect of an anatomical restriction may be augmented by a resonant standing wave created through reflection and convergence of transmitted arterial pressure waves along the vein [43]. The result is a localized increase in intravascular pressure at the venous end of the capillary bed (local hypertension), which, coupled with pulsatile shear stress and cyclic strain of the vessel wall can result in rupture of endothelial tight junctions and BBB breakdown [44]. Depending on magnitude of the latter, leakage of intravascular fluid, proteins and blood cells could occur. This would lead to significant changes in neuronal microenvironment, increased ISF and lymph production, local activation of the inflammatory cascade, iron deposition, oligodendrocyte loss, attraction of phagocytes, demyelination and eventually plaque formation [44].

Increased pressure at the venous end of a capillary bed has also important consequences on organ perfusion pressure and lymph production. According to Darcy's law, the rate of blood flow in a vessel of specific length is proportional to the pressure difference between its ends. Increased venous capillary pressure in the face of normal arterial capillary pressure would lead to hypoperfusion [43] and "virtual hypoxia" [45], both of which have been described in the setting of MS.

Increased filtration pressure due to venous hypertension and intravascular fluid and protein leakage due to BBB breakdown can lead to increases in interstitial fluid. Any factor that increases interstitial fluid also increases lymph flow [46]. Lymphatic drainage of the CNS regulates the balance of ISF and solutes within the CNS microenvironment and represents an accessory route through which excess fluid and proteins can flow from the interstitial spaces back into the blood. It also plays an important role in neuroimmunological reactions, through physiological drainage of antigens from the brain to regional lymph nodes, mostly cervical and lumbar [47]. In other words, altered lymph flow due to increased production or decreased outflow may affect both neuronal milieu and immune response.

The immune approach: Unlike most other organs, the CNS lacks an anatomically well-defined lymphatic system. Cerebrospinal fluid (CSF) 
Citation: Tsamopoulos NG, Kalodimou VE, and Vlachos S (2014) Chronic Cerebrospinal Venous Insufficiency in Multiple Sclerosis: The Hydrostatic-Immune Paradigm and the Flow Cytometry as a Diagnostic Tool. J Mult Scler 1: 103. doi:10.4172/jmso.1000103

Page 3 of 7

and ISF drainage pathways are different in humans but both drain partly or wholly to regional lymph nodes.

CSF production and outflow through the arachnoid villi and granulations to the venous sinuses has been extensively studied in the past. Even though it is the primary drainage route in humans [48,49], a small fraction of CSF drains through the cribriform plate into the nasal submucosa and ultimately to cervical lymph nodes [50].

ISF and solutes can initially diffuse through brain parenchyma, drain out of the brain along basement membranes of capillaries and cerebral arteries [51] and finally leave the artery walls and drain to adjacent cervical lymph nodes at the base of the skull [52].

The motive force for drainage along artery walls in a direction opposite to blood flow may be related to the pulsations of arteries and the creation of a contrary reflection wave [53]. A decrease in the amplitude of the contrary wave due to changes in arterial wall elasticity or other reasons could result in impediment of perivascular lymphatic drainage [53].

This unique anatomic arrangement, coupled with the presence of the blood brain barrier and the low expression of Major Histocompatibility Complex class II (MHC II) molecules creates an optimally sheltered environment and contributes to what is termed "immunological privilege" of the CNS [54].

Immune surveillance in the CNS differs somewhat from that of other organs, contributing to the "immune privilege". It consists of an afferent and an efferent arm.

The afferent arm involves antigen transfer and presentation to naive lymphocytes in regional lymph nodes or the spleen, resulting in their priming and activation. Antigen can be transferred by a fluid or a cellular route [55]. Soluble antigens (in ISF or CSF) can follow the fluid route through perivascular spaces [50-52] or along cranial (olfactory, optic, trigeminal, acoustic) and spinal nerves to regional lymph nodes [56]. CSF antigens can also gain direct access to the systemic circulation and to the spleen by absorption in arachnoid villi and granulations [47].

The cellular route involves antigen transfer by specialized antigen presenting cells (APCs) such as macrophages or dendritic cells (DCs). Macrophages containing myelin antigens have been described within CLNs of patients with MS [12]. This suggests that either APCs containing antigen migrate from the CNS to regional lymph nodes or that antigens draining from the CNS are taken up by APCs within the lymph nodes themselves [57]. To this date, there is no convincing evidence that inflammatory cells containing antigens can exit the healthy CNS parenchyma [55,58], even though this cannot be ruled out in the setting of inflammation.

Peripheral stimulation in lymphoid organs is another unresolved issue. Dendritic cells and macrophages containing myelin antigens have been found in CLNs, close to T-cells, suggesting interaction [12]. $\mathrm{T}$ cell responses to CNS antigens may depend on where this interaction takes place. Within CNS-draining CLNs, constitutive drainage of antigen from the healthy CNS can result in suppression of CNS-specific naïve T cell priming and induction of tolerance [59]. On the other hand, $\mathrm{T}$ cell responses initiated within peripheral, non-CNS draining lymph nodes, in response to systemic CNS antigen exposure, leads to $\mathrm{T}$ cell activation which eclipses the basal tolerogenic effect of soluble antigen drainage to CLNs [57].
The efferent arm involves inflammatory cell (monocytes, B- and Tcells) migration to the CNS and initiation of the inflammatory response. The process of cell activation and CNS homing is poorly understood. Cervical lymph node drainage may enhance CNS specificity of $\mathrm{T}$ cells by providing these cells with a CNS "homing beacon" in the form of signature molecules, such as CCR7 and CD68 [60]. Crossing of the BBB occurs by endothelial transmigration, similarly to what happens in other tissues: a sequential process of cellular rolling, adhesion and diapedesis, mediated and guided by adhesion molecules and chemokines [61]. Astrocytes are involved in this process, modulating BBB permeability and affecting lymphocyte recruitment [62]. At least three different routes of cell entry into the CNS have been described [63] but the fate of lymphocytes and macrophages once within the brain parenchyma is still controversial. They may remain in perivascular spaces, migrate through the basement membrane of the perivascular glia limitans into the CNS parenchyma and interact with target antigens [64] or simply undergo apoptosis [65].

Once inside the brain parenchyma, it is not clear how antigen presentation occurs in the absence of professional APCs and in the face of low expression of MHC II molecules [66]. T lymphocytes that have been pre-activated peripherically may release pro-inflammatory cytokines (IFN-c, TNF-a) and induce MHCII molecules in CNS cells [67]. Alternatively, sentinel monocytes/macrophages stationed in perivascular spaces may act as APCs [66], thus initiating autoimmunity and closing the circle of immune surveillance.

Auto-reactive T and B cells are fundamental for development of MS and require antigenic presentation of myelin antigens by APCs in order differentiate into effector cells [68]. In the initial stages of the disease, before lymphoid neogenesis occurs and tertiary lymphoid structures within the meningeal compartment are able to sustain a continuous inflammatory process [69], these interactions probably take place in CLNs [3] and are dependent on CNS antigen transport to those sites [57]. So, CLNs may act as loci of antigen presentation and T- or B-lymphocyte priming [54] but also as sites of tolerance induction towards CNS antigens [70,71]. In this case, alterations in lymph flow towards CLNs could theoretically lead to alterations in antigen presentation and processing with unknown consequences for CNS immune surveillance.

The combined hydrostatic-immune paradigm of CCSVI: Current data does not support the view that CCSVI is the sole cause of MS. However a wider role in MS pathogenesis cannot, at the present time, be confirmed or refuted. If CCSVI does correlate with MS, then alterations in CNS venous outflow must somehow be associated with induction or attenuation of inflammatory and immune responses. The only direct link between the vascular and immune systems is the lymphatic system.

We have described previously how the presence of venous stenoses (CCSVI) may lead to changes in venous outflow, which may in turn affect central nervous system (CNS) interstitial fluid (ISF) production and composition. The latter would also affect lymph production and outflow, altering CNS antigen presentation to regional lymph nodes. Altered antigen presentation could lead to altered immune responses in the form of increased autoimmunity or decreased tolerance to selfantigens (Figure 1). 
Citation: Tsamopoulos NG, Kalodimou VE, and Vlachos S (2014) Chronic Cerebrospinal Venous Insufficiency in Multiple Sclerosis: The Hydrostatic-Immune Paradigm and the Flow Cytometry as a Diagnostic Tool. J Mult Scler 1: 103. doi:10.4172/jmso.1000103

Page 4 of 7

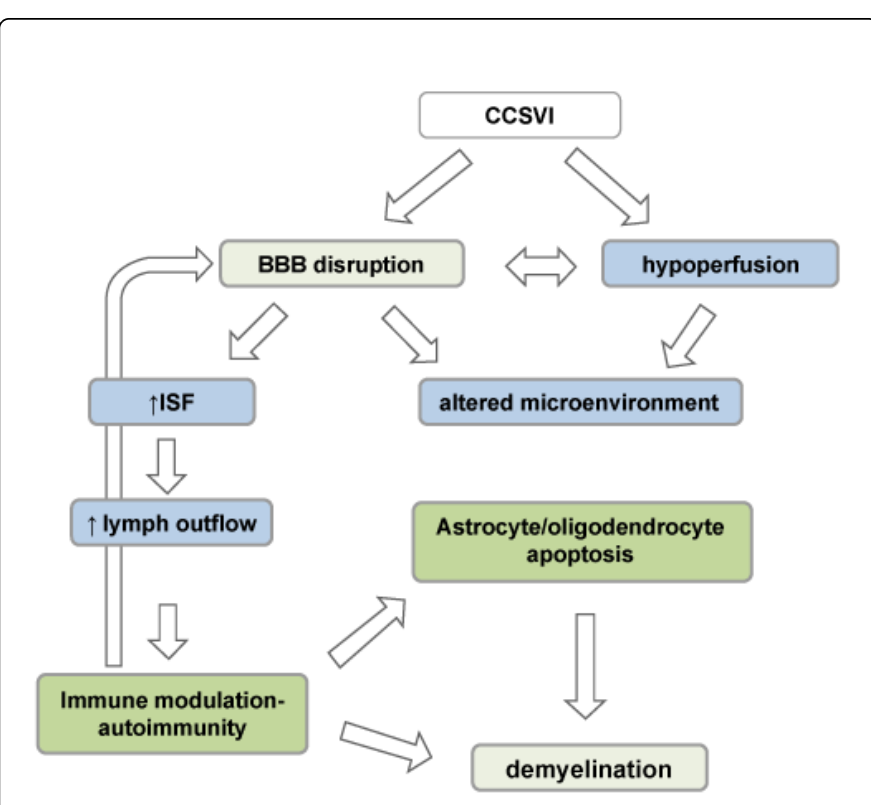

Figure 1: Interrelations of factors in the combined hydrostaticimmune paradigm of CCSVI. In blue: hydrostatic factors, in green: immune factors, in grey: mixed hydrostatic and immune factors.

More specifically, according to this theory, the presence of anatomic or functional venous stenoses leads to absolute or relative (functional) capillary venous hypertension due to reflected pressure waves. This results in dysfunction of tight junctions, perivenous edema and compromised $\mathrm{BBB}$ function, affecting parenchymal homeostasis and causing astrocyte and oligodendrocyte dysfunction. Local phagocytotic mechanisms activated by the death of these cells initiate demyelination. At the same time, increased ISF and lymph production coupled with an increased antigen load from apoptotic cells result in augmented antigen presentation to T-lymphocytes in CLNs, overwhelming their inherent tolerizing capacity and kick starting autoimmunity. This fuels the inflammatory process and perpetuates myelin damage.

Treatment of venous abnormalities (liberation procedure) would attenuate this process by alleviating venous hypertension and BBB damage. This would remove at least one of the hypothetical factors contributing towards astrocyte and/or oligodendrocyte apoptosis. The decreased perfusion pressure would also result in decreased ISF and lymph production and ultimately in decreased antigen transport and presentation to regional lymph nodes. In the initial stages of the disease, before the autoimmune process becomes compartmentalized in the Virchow-Robin spaces, altered antigen flow could lead to altered immune responses.

\section{Implications for research}

Unfortunately, study of the human lymphatic system is hampered by the difficulty to readily image it. Older techniques such as lymphangiography required considerable technical skill and were associated with complications. Newer, water soluble contrast agents minimize these problems but because of the difficulties in delivering sufficient amounts of agents necessary to produce X-ray contrast, classic lymphangiography is not routinely used today [72].
Lymphoscintigraphy is another imaging technique that enables twodimensional planar visualization of the lymphatic network after injection of Tc-99m-labelled colloid, unfortunately with a low spatial resolution [73]. Magnetic Resonance Imaging (MRI) of the lymphatic system (MR-lymphography) is easier to perform and does not require exposure to ionizing radiation. It involves interstitial or intravenous injection of gadolinium-labeled contrast agents or iron oxide particles and provides a very high spatial resolution [74]. The most promising imaging modality today is through optical imaging of the lymphatics. It is based upon the administration of contrast agents (fluorescein, indocyanine green) that, when excited by light of specific wavelength, exhibit fluorescence. This technique has been used successfully in mapping human lymphatics [75].

To this day, there have been no studies in MS patients regarding CNS lymph outflow or its changes after angioplasty treatment for CCSVI (liberation treatment). Nevertheless, reversal of abnormal lymphoscintigraphy after placement of venous stents for correction of associated venous obstruction in the lower limbs of MS patients has been reported [76], supporting the hydrostatic aspect of our theory.

It would be interesting to further explore whether lymph production and antigenic composition is different in MS patients with or without CCSVI and whether liberation treatment causes a change in these parameters. However, studies like that would have to involve injection of contrast agent directly into the brain parenchyma, something which is currently ethically unacceptable in humans. Extracranial lymph outflow or changes in CSF flow are much easier to image and could be used instead, but no such studies have been conducted and it is not known whether they accurately reflect intracranial lymph flow. Future improvements in lymphatic system imaging technique may provide this opportunity.

\section{Flow Cytometry as a diagnostic tool}

We could investigate the possible prognostic value of ploidy in humans and the disruptions occurring inside the cell cycle with flow cytomety as a diagnostic tool. Flow cytometry enables rapid quantification of DNA content of individual cells, and the cellular DNA content provides useful information about the ploidy, expressing the modal DNA value, and the proliferative activity in a tissue. The ability of flow cytometry to estimate cellular DNA content is based on the measurement of fluorescence from dyes which bind in a stoichiometric manner to DNA [77].

As the DNA content is duplicated prior to cell division, mathematical models have been derived which can estimate the percentage of cells in different phases of the cell cycle. The use of flow cytometry for DNA analysis between family members with genetically linked diseases provides fast results, permits multiparameter analysis correlating DNA content with antigen expression, and also provides sensitivity for detecting near-diploid aneuploid peaks [78].

In cases like this, we consider that a genetic profile analysis should be performed to patients and their first degree relatives for more efficient therapies and follow ups of the disease. Flow Cytometry as an analysis method is rapid, sensitive, accurate and efficient for the genetic analysis and the progress of the disease.

\section{Conclusions}

MS is considered a primarily autoimmune disease but this paradigm is slowly shifting towards a combined autoimmune- 
Citation: Tsamopoulos NG, Kalodimou VE, and Vlachos S (2014) Chronic Cerebrospinal Venous Insufficiency in Multiple Sclerosis: The Hydrostatic-Immune Paradigm and the Flow Cytometry as a Diagnostic Tool. J Mult Scler 1: 103. doi:10.4172/jmso.1000103

Page 5 of 7

neurodegenerative model [79]. The pathogenetic mechanism is almost certainly multi-factorial and even though the initial trigger is still unknown, some of the waypoints on the road leading to demyelination and axonal loss have been discovered. It is not clear yet to what extend venous outflow abnormalities contribute to this process. Altered neuronal microenvironment and changes in antigen presentation to regional lymph nodes may provide a theoretical framework combining hydrostatic and immune effects. Lymph nodes are a site of sensitive immune regulation where both immune suppression and immune activation take place $[80,81]$ CCSVI and its treatment may act via tipping the balance towards one or the other side of the scales.

\section{References}

1. Weinshenker BG, Bass B, Rice GP, Noseworthy J, Carriere W, et al. (1989) The natural history of multiple sclerosis: a geographically based study. 2. Predictive value of the early clinical course. Brain 112 : 1419-1428.

2. Moore GR (2010) Current concepts in the neuropathology and pathogenesis of multiple sclerosis. Can J Neurol Sci 37 Suppl 2: S5-15.

3. Ontaneda D, Hyland M, Cohen JA (2012) Multiple sclerosis: new insights in pathogenesis and novel therapeutics. Annu Rev Med 63: 389-404.

4. Charcot J (1868) Histology of "sclerose en plaque" Gazette Hosp 41: 554-66.

5. Zamboni P, Menegatti E, Galeotti R, Malagoni AM, Tacconi G, et al. (2009) The value of cerebral Doppler venous haemodynamics in the assessment of multiple sclerosis. J Neurol Sci 282: 21-27.

6. Zamboni P (2006) The big idea: iron-dependent inflammation in venous disease and proposed parallels in multiple sclerosis. J R Soc Med 99: 589-593.

7. Zamboni P, Galeotti R, Menegatti E, Malagoni AM, Tacconi G, et al. (2009) Chronic cerebrospinal venous insufficiency in patients with multiple sclerosis. J Neurol Neurosurg Psychiatry 80: 392-399.

8. Ghezzi A, Comi G, Federico A (2011) Chronic cerebro-spinal venous insufficiency (CCSVI) and multiple sclerosis. Neurol Sci 32: 17-21.

9. Thapar A, Lane T, Nicholas R, Friede T, Ellis M, et al. (2011) Systematic review of sonographic chronic cerebrospinal venous insufficiency findings in multiple sclerosis. Phlebology 26: 319-325.

10. Barnett $\mathrm{MH}$, Prineas JW (2004) Relapsing and remitting multiple sclerosis: pathology of the newly forming lesion. Ann Neurol 55: 458-468.

11. Barnett $\mathrm{MH}$, Sutton I (2006) The pathology of multiple sclerosis: a paradigm shift. Curr Opin Neurol 19: 242-247.

12. de Vos AF, van Meurs M, Brok HP, Boven LA, Hintzen RQ, et al. (2002) Transfer of central nervous system autoantigens and presentation in secondary lymphoid organs. J Immunol 169: 5415-5423.

13. Fabriek BO, Zwemmer JN, Teunissen CE, Dijkstra CD, Polman CH, et al. (2005) In vivo detection of myelin proteins in cervical lymph nodes of MS patients using ultrasound-guided fine-needle aspiration cytology. J Neuroimmunol 161: 190-194.

14. Fischer HG, Reichmann G (2001) Brain dendritic cells and macrophages/ microglia in central nervous system inflammation. J Immunol 166: 2717-2726.

15. Lucchinetti C, Brück W, Parisi J, Scheithauer B, Rodriguez M, et al. (2000) Heterogeneity of multiple sclerosis lesions: implications for the pathogenesis of demyelination. Ann Neurol 47: 707-717.

16. De Keyser J, Steen C, Mostert JP, Koch MW (2008) Hypoperfusion of the cerebral white matter in multiple sclerosis: possible mechanisms and pathophysiological significance. J Cereb Blood Flow Metab 28: 1645-1651.

17. Blinkenberg M, Rune K, Jensen CV, Ravnborg M, Kyllingsbaek S, et al. (2000) Cortical cerebral metabolism correlates with MRI lesion load and cognitive dysfunction in MS. Neurology 54: 558-564.

18. Van Au Duong M, Audoin B, Le Fur Y, Confort-Gouny S, Malikova I, et al. (2007) Relationships between gray matter metabolic abnormalities and white matter inflammation in patients at the very early stage of MS : a MRSI study. J Neurol 254: 914-923.

19. Law M, Saindane AM, Ge Y, Babb JS, Johnson G, et al. (2004) Microvascular abnormality in relapsing-remitting multiple sclerosis: perfusion MR imaging findings in normal-appearing white matter. Radiology 231: 645-652.

20. Adhya S, Johnson G, Herbert J, Jaggi H, Babb JS, et al. (2006) Pattern of hemodynamic impairment in multiple sclerosis: dynamic susceptibility contrast perfusion MR imaging at 3.0 T. Neuroimage 33: 1029-1035.

21. Inglese M, Park SJ, Johnson G, Babb JS, Miles L, et al. (2007) Deep gray matter perfusion in multiple sclerosis: dynamic susceptibility contrast perfusion magnetic resonance imaging at 3 T. Arch Neurol 64: 196-202.

22. Saindane AM, Law M, Ge Y, Johnson G, Babb JS, et al. (2007) Correlation of diffusion tensor and dynamic perfusion MR imaging metrics in normal-appearing corpus callosum: support for primary hypoperfusion in multiple sclerosis. AJNR Am J Neuroradiol 28: 767-772.

23. Aboul-Enein F, Lassmann H (2005) Mitochondrial damage and histotoxic hypoxia: a pathway of tissue injury in inflammatory brain disease? Acta Neuropathol 109: 49-55.

24. Filosa JA, Bonev AD, Nelson MT (2004) Calcium dynamics in cortical astrocytes and arterioles during neurovascular coupling. Circ Res 95: e73-81.

25. Metea MR, Newman EA (2006) Glial cells dilate and constrict blood vessels: a mechanism of neurovascular coupling. J Neurosci 26: 2862-2870.

26. Zeinstra E, Wilczak N, De Keyser J (2000) [3H]dihydroalprenolol binding to beta adrenergic receptors in multiple sclerosis brain. Neurosci Lett 289: 75-77.

27. Lightman S, McDonald WI, Bird AC, Francis DA, Hoskins A, et al. (1987) Retinal venous sheathing in optic neuritis. Its significance for the pathogenesis of multiple sclerosis. Brain 110 : 405-414.

28. Ge Y, Law M, Herbert J, Grossman RI (2005) Prominent perivenular spaces in multiple sclerosis as a sign of perivascular inflammation in primary demyelination. AJNR Am J Neuroradiol 26: 2316-2319.

29. Tallantyre EC, Brookes MJ, Dixon JE, Morgan PS, Evangelou N, et al. (2008) Demonstrating the perivascular distribution of MS lesions in vivo with 7-Tesla MRI. Neurology 70: 2076-2078.

30. Ge Y, Zohrabian VM, Grossman RI (2008) Seven-Tesla magnetic resonance imaging: new vision of microvascular abnormalities in multiple sclerosis. Arch Neurol 65: 812-816.

31. Prineas J (1975) Pathology of the early lesion in multiple sclerosis. Hum Pathol 6: 531-554.

32. Lucchinetti CF, Brueck W, Rodriguez M, Lassmann H (1998) Multiple sclerosis: lessons from neuropathology. Semin Neurol 18: 337-349.

33. Adams CW (1988) Perivascular iron deposition and other vascular damage in multiple sclerosis. J Neurol Neurosurg Psychiatry 51: 260-265.

34. Shaw PJ, Smith NM, Ince PG, Bates D (1987) Chronic periphlebitis retinae in multiple sclerosis. A histopathological study. J Neurol Sci 77: 147-152.

35. Sinnecker T, Bozin I, Dörr J, Pfueller CF, Harms L, et al. (2013) Periventricular venous density in multiple sclerosis is inversely associated with T2 lesion count: a 7 Tesla MRI study. Mult Scler 19: 316-325.

36. Zivadinov R, Poloni GU, Marr K, Schirda CV, Magnano CR, et al. (2011) Decreased brain venous vasculature visibility on susceptibility-weighted imaging venography in patients with multiple sclerosis is related to chronic cerebrospinal venous insufficiency. BMC Neurol 11: 128.

37. Wuerfel J, Paul F, Zipp F (2007) Cerebral blood perfusion changes in multiple sclerosis. J Neurol Sci 259: 16-20.

38. Kostyk SK, Kowall NW, Hauser SL (1989) Substance P immunoreactive astrocytes are present in multiple sclerosis plaques. Brain Res 504: 284-288.

39. Blamire AM, Anthony DC, Rajagopalan B, Sibson NR, Perry VH, et al. (2000) Interleukin-1beta -induced changes in blood-brain barrier 
Citation: Tsamopoulos NG, Kalodimou VE, and Vlachos S (2014) Chronic Cerebrospinal Venous Insufficiency in Multiple Sclerosis: The Hydrostatic-Immune Paradigm and the Flow Cytometry as a Diagnostic Tool. J Mult Scler 1: 103. doi:10.4172/jmso.1000103

Page 6 of 7

permeability, apparent diffusion coefficient, and cerebral blood volume in the rat brain: a magnetic resonance study. J Neurosci. 20: 8153-8159.

40. Sibson NR, Blamire AM, Perry VH, Gauldie J, Styles P, et al. (2002) TNFalpha reduces cerebral blood volume and disrupts tissue homeostasis via an endothelin- and TNFR2-dependent pathway. Brain 125: 2446-2459.

41. Claudio L, Raine CS, Brosnan CF (1995) Evidence of persistent bloodbrain barrier abnormalities in chronic-progressive multiple sclerosis. Acta Neuropathol 90: 228-238.

42. Plumb J, McQuaid S, Mirakhur M, Kirk J (2002) Abnormal endothelial tight junctions in active lesions and normal-appearing white matter in multiple sclerosis. Brain Pathol 12: 154-169.

43. Tucker TW (2011) A physics link between venous stenosis and multiple sclerosis. Med Hypotheses 77: 1074-1078.

44. Talbert DG (2008) Raised venous pressure as a factor in multiple sclerosis. Med Hypotheses 70: 1112-1117.

45. Zamboni P, Menegatti E, Weinstock-Guttman B, Dwyer MG, Schirda CV, et al. (2011) Hypoperfusion of brain parenchyma is associated with the severity of chronic cerebrospinal venous insufficiency in patients with multiple sclerosis: a cross-sectional preliminary report. BMC Med 9: 22.

46. Guyton AC, Hall JE (2006) Textbook of medical physiology (11th edn) Elsevier Saunders; Philadelphia, pp- 192.

47. Weller RO, Djuanda E, Yow HY, Carare RO (2009) Lymphatic drainage of the brain and the pathophysiology of neurological disease. Acta Neuropathol 117: 1-14.

48. Davson H, Welch K, Segal MB (1987) Physiology and pathophysiology of the cerebrospinal fluid. Churchill Livingstone, Edinburgh.

49. Johanson CE, Duncan JA 3rd, Klinge PM, Brinker T, Stopa EG, et al. (2008) Multiplicity of cerebrospinal fluid functions: New challenges in health and disease. Cerebrospinal Fluid Res 5: 10.

50. Johnston M, Zakharov A, Papaiconomou C, Salmasi G, Armstrong D (2004) Evidence of connections between cerebrospinal fluid and nasal lymphatic vessels in humans, non-human primates and other mammalian species. Cerebrospinal Fluid Res 1:2.

51. Carare RO, Bernardes-Silva M, Newman TA, Page AM, Nicoll JA, et al. (2008) Solutes, but not cells, drain from the brain parenchyma along basement membranes of capillaries and arteries: significance for cerebral amyloid angiopathy and neuroimmunology. Neuropathol Appl Neurobiol 34: 131-144.

52. Clapham R, O'Sullivan E, Weller RO, Carare RO (2010) Cervical lymph nodes are found in direct relationship with the internal carotid artery: significance for the lymphatic drainage of the brain. Clin Anat 23: 43-47.

53. Schley D, Carare-Nnadi R, Please CP, Perry VH, Weller RO (2006) Mechanisms to explain the reverse perivascular transport of solutes out of the brain. J Theor Biol 238: 962-974.

54. Romo-González T, Chavarría A, Pérez-H J (2012) Central nervous system: a modified immune surveillance circuit? Brain Behav Immun 26: 823-829.

55. Galea I, Bechmann I, Perry VH (2007) What is immune privilege (not)? Trends Immunol 28: 12-18.

56. Cserr HF, Harling-Berg CJ, Knopf PM (1992) Drainage of brain extracellular fluid into blood and deep cervical lymph and its immunological significance. Brain Pathol 2: 269-276.

57. Weller RO, Galea I, Carare RO, Minagar A (2010) Pathophysiology of the lymphatic drainage of the central nervous system: Implications for pathogenesis and therapy of multiple sclerosis. Pathophysiology 17: 295-306.

58. Hatterer E, Davoust N, Didier-Bazes M, Vuaillat C, Malcus C, et al. (2006) How to drain without lymphatics? Dendritic cells migrate from the cerebrospinal fluid to the B-cell follicles of cervical lymph nodes. Blood 107: 806-812.

59. Kraal G, Samsom JN, Mebius RE (2006) The importance of regional lymph nodes for mucosal tolerance. Immunol Rev 213: 119-130.

60. Kivisäkk P, Mahad DJ, Callahan MK, Sikora K, Trebst C, et al. (2004) Expression of CCR7 in multiple sclerosis: implications for CNS immunity. Ann Neurol 55: 627-638.
61. Drevets DA, Leenen PJ (2000) Leukocyte-facilitated entry of intracellular pathogens into the central nervous system. Microbes Infect 2: 1609-1618.

62. Hickey WF (2001) Basic principles of immunological surveillance of the normal central nervous system. Glia 36: 118-124.

63. Ransohoff RM, Kivisäkk P, Kidd G (2003) Three or more routes for leukocyte migration into the central nervous system. Nat Rev Immunol 3: 569-581.

64. Bechmann I, Galea I, Perry VH (2007) What is the blood-brain barrier (not)?. Trends Immunol 28: 5-11.

65. Hochmeister S, Zeitelhofer M, Bauer J, Nicolussi EM, Fischer MT, et al. (2008) After injection into the striatum, in vitro-differentiated microgliaand bone marrow-derived dendritic cells can leave the central nervous system via the blood stream. Am J Pathol 173: 1669-1681.

66. Neumann H (2001) Control of glial immune function by neurons. Glia 36: 191-199.

67. Carson MJ, Thrash JC, Walter B (2006) The cellular response in neuroinflammation: The role of leukocytes, microglia and astrocytes in neuronal death and survival. Clin Neurosci Res 6: 237-245.

68. Glass CK, Saijo K, Winner B, Marchetto MC, Gage FH (2010) Mechanisms underlying inflammation in neurodegeneration. Cell 140: 918-934.

69. Corcione A, Casazza S, Ferretti E, Giunti D, Zappia E, et al. (2004) Recapitulation of B cell differentiation in the central nervous system of patients with multiple sclerosis. Proc Natl Acad Sci U S A 101: 11064-11069.

70. Wolvers DA, Coenen-de Roo CJ, Mebius RE, van der Cammen MJ, Tirion F, et al. (1999) Intranasally induced immunological tolerance is determined by characteristics of the draining lymph nodes: studies with OVA and human cartilage gp-39. J Immunol 162: 1994-1998.

71. Wenkel H, Streilein JW, Young MJ (2000) Systemic immune deviation in the brain that does not depend on the integrity of the blood-brain barrier. J Immunol 164: 5125-5131.

72. Sharma R, Wendt JA, Rasmussen JC, Adams KE, Marshall MV, et al. (2008) New horizons for imaging lymphatic function. Ann N Y Acad Sci 1131: 13-36.

73. Szuba A, Shin WS, Strauss HW, Rockson S (2003) The third circulation: radionuclide lymphoscintigraphy in the evaluation of lymphedema. J Nucl Med 44: 43-57.

74. Misselwitz B (2006) MR contrast agents in lymph node imaging. Eur J Radiol 58: 375-382.

75. Unno N, Inuzuka K, Suzuki M, Yamamoto N, Sagara D, et al. (2007) Preliminary experience with a novel fluorescence lymphography using indocyanine green in patients with secondary lymphedema. J Vasc Surg 45: 1016-1021.

76. Raju S, Owen S Jr, Neglen P (2001) Reversal of abnormal lymphoscintigraphy after placement of venous stents for correction of associated venous obstruction. J Vasc Surg 34: 779-784.

77. Dressier LG (1990) Controls, standards, and histogram interpretation in DNA flow cytometry. Academic Press, San Diego, California 33: 157-171.

78. Kalodimou VE (2013) Basic Principles in Flow Cytomety, Bethesda Md AABB.

79. Stadelmann C (2011) Multiple sclerosis as a neurodegenerative disease: pathology, mechanisms and therapeutic implications. Curr Opin Neurol 24: 224-229.

80. van Zwam M, Huizinga R, Melief MJ, Wierenga-Wolf AF, van Meurs M, et al. (2009) Brain antigens in functionally distinct antigen-presenting cell populations in cervical lymph nodes in MS and EAE. J Mol Med (Berl) 87: 273-286

81. Boven LA, Van Meurs M, Van Zwam M, Wierenga-Wolf A, Hintzen RQ, et al. (2006) Myelin-laden macrophages are anti-inflammatory, consistent with foam cells in multiple sclerosis. Brain 129: 517-526. 